\title{
Interactions within the Hand Representation in Primary Somatosensory Cortex of Primates
}

\author{
Michael L. Lipton, ${ }^{1,2,3,4}$ Mark C. Liszewski, ${ }^{8}$ M. Noelle 0'Connell, ${ }^{5}$ Aimee Mills, ${ }^{5}$ John F. Smiley, ${ }^{5}$ Craig A. Branch, ${ }^{1,2}$ \\ Joseph R. Isler, ${ }^{7}$ and Charles E. Schroeder ${ }^{5,6}$ \\ ${ }^{1}$ The Gruss Magnetic Resonance Research Center, Departments of ${ }^{2}$ Radiology, ${ }^{3}$ Psychiatry and Behavioral Sciences, and The Dominick P. Purpura \\ Department of Neuroscience, Albert Einstein College of Medicine, Bronx, New York 10461, ${ }^{4}$ Department of Radiology, Montefiore Medical Center, Bronx, \\ New York 10467, ${ }^{5}$ Program in Cognitive Neuroscience and Schizophrenia, The Nathan S. Kline Institute for Psychiatric Research, Orangeburg, New York \\ 10962, Departments of ${ }^{6}$ Psychiatry and ${ }^{7}$ Pediatrics, Columbia University College of Physicians and Surgeons, New York, New York 10032, and ${ }^{8}$ Department \\ of Radiology, Brigham and Women’s Hospital, Boston, Massachusetts 02115
}

Previous studies indicate that primary somatosensory cortical area $3 \mathrm{~b}$ in macaques contains a somatotopic map of the hand, encompassing representations of each digit. However, numerous observations including recent findings in anesthetized New World monkeys indicate that that the digit representations within the map are not discrete. We assessed the generality and spatial extent of these effects in awake macaques. We show that, within a given digit representation, (1) there is response to stimulation of all other digits tested, extending across most or all of the digit map, and (2) response to stimulation of the locally preferred digit is modulated by concurrent stimulation of each of the other digits. Control experiments rule out effects of attention and mechanical spread of stimulation. We thus confirm that, even at the first level of somatosensory cortical processing, inputs from potentially all of the digits frame the context within which the input to a single digit is represented.

\section{Introduction}

Studies of the primate hand representation in primary somatosensory cortical area $3 \mathrm{~b}$ have consistently identified separate representations of each digit (Powell and Mountcastle, 1959b; Jones and Powell, 1969; Paul et al., 1972; Sur et al., 1982; Pons et al., 1987; Kaas, 1993). This detailed surface representation is a cornerstone of hierarchical organization in the somatosensory system, providing a substrate for specific computational functions of higher order cortices (Burton and Sinclair, 1996; Moore et al., 1999). The capacity for radical spatial reorganization in the area $3 \mathrm{~b}$ digit map shows clearly that it is not invariant (Allard et al., 1991; Rasmusson et al., 1992; Mogilner et al., 1993; Zarzecki et al., 1993; Schroeder et al., 1997). The rapidity and "preferred pattern" of heterotopic functional reorganization in area 3b (Allard et al., 1991; Zarzecki et al., 1993) suggests changes in the gain of existing heterotopic neuronal connections underpin these effects (Jenkins and Merzenich, 1987; Turnbull and Rasmusson, 1990; Clarey et al., 1996; Coq and Xerri, 1999). The existence of heterotopic connections in the normal intact digit map can be inferred from numerous previous observations in monkeys. For example, (1) the axon terminals of single thalamocortical neurons span

Received Sept. 9, 2009; revised Aug. 27, 2010; accepted Aug. 30, 2010.

This work was supported in part by National Institutes of Health Grant K08-MH67082 (M.L.L.) and National Science Foundation Grant NSF-0519410 (C.E.S.).

Correspondence should be addressed to either of the following: Dr. Michael L. Lipton, The Gruss Magnetic Resonance Center, Albert Einstein College of Medicine, 1300 Morris Park Avenue, Bronx, NY 10461, E-mail: michael.lipton@einstein.yu.edu; or Dr. Charles E. Schroeder, The Nathan S. Kline Institute for Psychiatric Research, 140 Old Orangeburg Road, Orangeburg, NY 10962, E-mail: schrod@nki.rfmh.org.

DOI:10.1523/JNEUROSCI.4765-09.2010

Copyright $\odot 2010$ the authors $\quad 0270-6474 / 10 / 3015895-09 \$ 15.00 / 0$ regions of area $3 \mathrm{~b}$ cortex several times the diameter of an individual digit representation (Garraghty and Sur, 1990), (2) stimulation of digits outside of the driving input of a single area $3 \mathrm{~b}$ neuron elicits "subthreshold" postsynaptic potentials (Zarzecki et al., 1993), (3) ambient activity in the "median nerve territory" of area $3 \mathrm{~b}$ is strongly modulated by stimulation of radial nerve afferents (Schroeder et al., 1995a), (4) the spatial pattern of hemodynamic response in area $3 \mathrm{~b}$ to single digit stimulation is changed by adjacent digit stimulation (Chen et al., 2003; Friedman et al., 2008), and perhaps most importantly (5) a recent study in anesthetized New World monkeys (Reed et al., 2008) showed that neurons at least $2 \mathrm{~mm}$ apart in the area $3 \mathrm{~b}$ hand map could synchronize stimulus-evoked firing.

Neuronal recordings in rodent barrel cortex (Nicolelis et al., 1998; Moore et al., 1999; Andermann and Moore, 2006), raccoon hand (Greek et al., 2003), and monkey forearm (Gardner and Costanzo, 1980), as well as magnetoencephalographic studies of the human hand representation (Buchner et al., 1999; Noppeney et al., 1999; Braun et al., 2000, 2002; Iguchi et al., 2001; Jones et al., 2007) indicate that area 3 b neurons integrate far more stimulus and "state of the system" information than initially suspected.

Collectively, the above findings suggest that expression of the digit map in primate area $3 \mathrm{~b}$ is dynamic and context dependent. We systematically tested functional interactions between digit representations to define the dimensions over which such heterotopic interactions occur in awake macaques. Our findings show that activity within a digit representation is modulated by stimulation of any of the other digits, and that interactions can extend across the entire digit map. Importantly, we demonstrate that these effects occur in real time in awake intact animals. Control 
experiments rule out effects attributable to attention and mechanical spread of stimulation. We thus confirm that, even at the first stage of somatosensory cortical processing, inputs from potentially all of the digits frame the context within which the input to a single digit is represented.

\section{Materials and Methods}

Intracortical multielectrode recordings were performed in two female rhesus macaques (Macaca mulatta). All animal experimentation was reviewed, approved, and monitored by the local Institutional Animal Care and Use Committee and complied with United States Public Health Service guidelines for animal research.

Surgical preparation. Animals were surgically prepared for chronic awake electrophysiological recording using standard methods (Schroeder et al., 1998). Preparatory surgery was performed under deep (1-3\%) isoflurane anesthesia. A titanium head post was fastened to the posterior skull using titanium orthopedic brackets and bone screws to allow painless restraint of the head during recording. Cilux plastic recording chambers (Crist Instrument Company) were positioned bilaterally over craniotomies exposing the region of the primary cortical hand representation at the lateral portion of the central sulcus. Each recording chamber was positioned to optimize the angle of penetration in area $3 \mathrm{~b}$ to the greatest extent possible and entailed orienting the chambers so that initial cortical penetration occurred at an angle of $\sim 60^{\circ}$, depending on the anatomy of each hemisphere in each animal. Care was taken to maintain the dura intact.

Behavioral preparation. After recovery from surgery, monkeys were accustomed to a primate chair and head restraint. They were not required to attend to or discriminate any of the stimuli, but rather, were explicitly habituated to both electrical and mechanical forms of somatosensory stimulation (see below).

Median nerve electrical stimulation. Two gold cup electrodes were positioned over the median nerve just proximal to the wrist and electrical stimulation from a GRASS S8 stimulator (Astro-Med) delivered a $200 \mu \mathrm{s}$ duration square-wave pulse at $0.5 \mathrm{~Hz}$. Stimulus intensity was titrated before each session to just subthreshold for the adductor pollicis brevis (APB) twitch. Stimulus intensity was monitored continuously (using the distal APB twitch) during recording.

Mechanical stimulation. Mechanical stimulation was delivered to $\mathrm{d} 2-\mathrm{d} 5$ of each hand using a 4D Neuroscience Somatosensory Stimulator (4D Neuroimaging). The stimulus generation components were located outside the sound-attenuated recording chamber to ensure that no auditory stimulus confounded the experiments. Because the stimulus device was limited to eight channels, we were not able to study $\mathrm{d} 1$ or other hand surfaces in this study. The pneumatically driven device was set to produce a $5 \mathrm{~ms}$ duration deflection of a flexible vinyl diaphragm positioned under each digit pad. The diaphragms were kept in position by incorporating them into fabric sheaths that were placed over the monkeys' fingers, and the hands and fingers were gently restrained. Under these conditions, the diaphragm provides a light tap to the digit without causing gross motion of the finger. Stimulus intensity is titrated to elicit a somatosensory evoked response in area $3 b$ that just exceeds 2 SDs from stimulus-free baseline. The animals were continuously monitored via closed-circuit video, and gross finger movement was not a significant problem. On the rare occasions in which gross movement occurred and degraded the recording quality, the problematic trials were discarded.

Strain gauge measurements. To eliminate artifacts caused by animal movement, these measurements were made with the animal under deep sedation. Ketamine $(15 \mathrm{mg} / \mathrm{kg})$ was administered intramuscularly with additional doses administered as needed to keep the animal motionless. Body temperature was maintained using a thermostatically controlled heating pad, and standard physiologic and visual monitoring was continued until the animal recovered from sedation. The stimulus device (see above, Mechanical stimulation) for each digit was attached to a dedicated high-fidelity pressure transducer (Gould P23 miniature pressure transducers; Harvard Apparatus) and each transducer was connected to a strain gauge amplifier (series 4600; Harvard Apparatus). Tubing was spliced with a "T" connector to permit concurrent pressure measurement. Transducers, amplifiers, and connector tubing were assured to have unity gain over the $0-500 \mathrm{~Hz}$ range. Each pressure channel was sampled at $200 \mathrm{~Hz}$, with a 16-bit $\mathrm{A} / \mathrm{D}$ and a pressure range of \pm 100 $\mathrm{mmHg}$. Pressures were recorded using a Pentium PC running LabView Biobench software (Vernier Software \& Technology). Voltage in the trigger channel was sampled with a voltage range of $\pm 10 \mathrm{~V}$. Thus, stimulation was applied to one digit, exactly as it was during the actual experiments, while pressure was recorded simultaneously at the stimulated as well as the adjacent digits.

Digit anesthesia experiments. Experiments were performed under general anesthesia (see below, Recording under general anesthesia) using both mechanical and electrical stimulation with the recording multielectrode in a confirmed area 3 b layer 4 location. For electrical stimulation, a pair of sterile needle electrodes was inserted, under sterile conditions, into the subcutaneous tissues of the medial and lateral surface of the digit tip. A conductive mesh was placed snugly around the proximal segment of the same digit, embedded in conductive gel, and connected to ground. This measure was taken to minimize any chance that the current delivered would conduct to other parts of the hand or to adjacent digits. After recording of responses to stimulation of the preferred and adjacent digits at a stimulus intensity (pressure or electric current) just suprathreshold for the stimulus evoked response (see description of stimulus calibration below), the preferred digit was anesthetized by infiltrating the tissues surrounding the digital nerves with a mixture ( $1 \mathrm{cc}$ total) of equal volumes of lidocaine $(1 \%)$ and bupivicaine $(0.25 \%)$. This combination of drugs provided rapid onset of a durable nerve block. The block remained fully effective (no detectable electrical somatosensory evoked response) for at least $12 \mathrm{~min}$ in each case. The evoked response increased slowly from that point and was at baseline after 15-20 min. Recording was repeated at the same intensity and at up to six times the stimulus intensity during the period of complete block. After recovery from local anesthesia, recordings were repeated at the immediately suprathreshold intensity used initially.

Data collection. Data were collected during penetrations of the hand representation in area $3 \mathrm{~b}$ with $0.34 \mathrm{~mm}$ diameter linear array multicontact electrodes (24 contacts; $0.1-0.3 \mathrm{M} \Omega$ impedence; Neurotrak) that record from all cortical layers simultaneously (Fig. 1). The multielectrodes used in this study had an intercontact spacing of $200 \mu \mathrm{m}$, which allowed concurrent sampling over a $5 \mathrm{~mm}$ span of brain tissue. Thus, we could completely bracket a single cortical region, even with a nonoptimal penetration angle. With an ideal penetration angle (i.e., orthogonal to the lamination pattern), this type of electrode could record from two adjacent cortical areas (e.g., areas 4 and $3 \mathrm{~b}$ or 1 and $3 \mathrm{~b}$ ) simultaneously. After preamplification $(10 \times)$ at the electrode headstage, signals from each channel were amplified $(1000 \times)$, bandpass filtered $(0.1 \mathrm{~Hz}$ to $3 \mathrm{kHz})$, and processed separately to extract field potential and action potential measures. Field potential profiles were obtained by digitizing the signal at $20 \mathrm{kHz}$ and averaging over 100 stimulus presentations. The spatial voltage gradient in the field potential profile was quantified using a threepoint formula for estimation of the second spatial derivative of voltage, the current source density (CSD) profile (Nicholson and Freeman, 1975; Schroeder et al., 1995b). As discussed below, the local field potential profile and its derivative CSD profile were used to help in positioning the electrode array, but the primary dependent measure was multiunit activity (MUA). MUA was obtained from the signal at each contact by highpass filtering the amplifier output at $500 \mathrm{~Hz}$ to isolate action potential frequency activity, full-wave rectifying the high-frequency activity, integrating the activity down to $1 \mathrm{kHz}$, and then averaging the single sweep responses $(n=100)$. Full-wave rectification ensures that we capture the full magnitude of the action potential response, without cancellation of negative and positive deflections. Our measure thus yields an estimate of the envelope firing pattern in local neurons (Legatt et al., 1980), measured in microvolts. Upward deflection represents an increase in activity, and downward deflection represents activity decrease relative to the prestimulus baseline.

Targeting the hand region of area $3 b$. We used several approaches to ensure that recordings were derived from area $3 \mathrm{~b}$. First, electrode penetrations were targeted based on high-resolution presurgical magnetic resonance imaging (MRI), and the recording chambers were centered over the lateral portion of the central sulcus, under direct visual control 

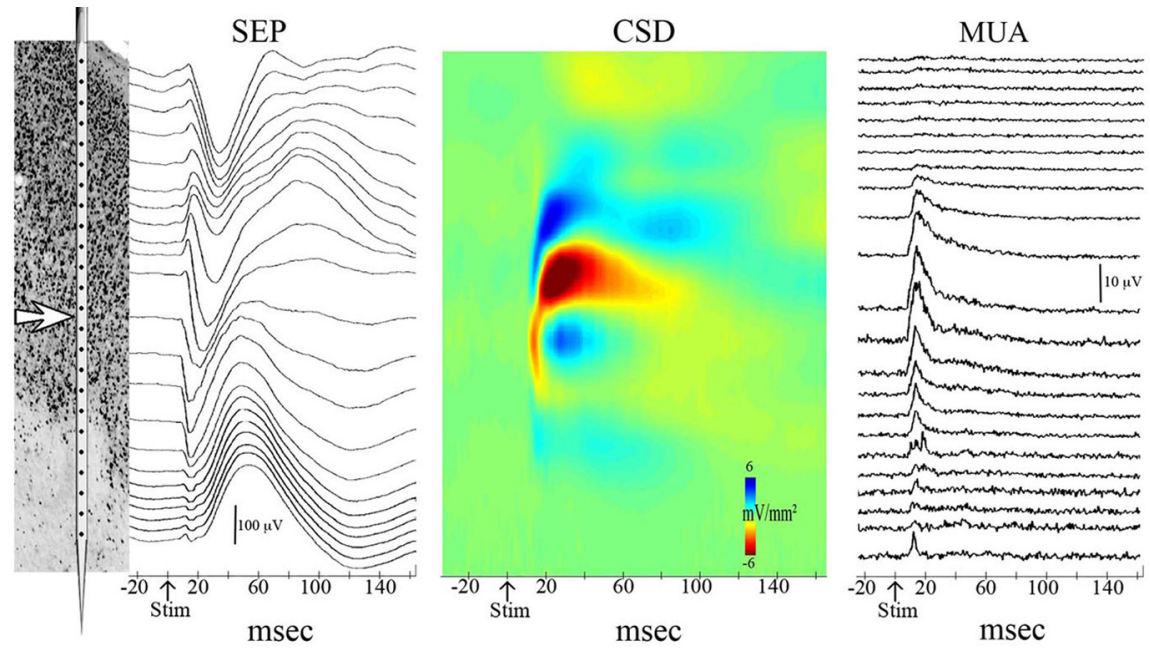

Figure 1. Laminar profile of cortical activity. A schematic drawing of the multicontact electrode is shown at far left, scaled and positioned with respect to a section of area $3 \mathrm{~b}$ as shown in Figure 2 . With the electrode approach angle used in this study, the electrode penetrates through the pial surface of area $3 \mathrm{~b}$ and is lowered so that the contacts completely bracket the cortical layers. Simultaneous recording of stimulus-evoked responses across channels yields the laminar field potential (left) and multiunit activity profiles (right). Field potential recordings from the top-most and bottom-most groups of contacts show minimal, mostly linear voltage gradients. Contacts at the central portion of the array record steep voltage gradients with inversion of the voltage profile indicating the interface between layers 3 and 4 (arrow). The second spatial derivative of voltage across channels yields the CSD profile, shown in the center. This display shows the laminar pattern of current sources (blue; net outward transmembrane current flow) and current sinks (red; net inward transmembrane current flow), yielding an index of the laminar pattern of synaptic activity. Early depolarization (current sink) is evident in layer 4, followed by hyperpolarization (current sources) in the extragranular layers. This pattern is characteristic of feedforward synaptic activity. Action potentials (MUA; at far right) show maximal amplitude centered in layer 4 and lower layer 3, consistent with the excitatory nature of the feedforward process. SEP, Stimulus-evoked potential.

during the implant surgery. Second, each subject underwent initial mapping penetrations to help define the position of the hand-responsive area in the posterior bank of the central sulcus, and this mapping was refined over the course of experimental penetrations. Third, hand responsive sites were considered as potentially within area $3 \mathrm{~b}$ only if they were found at a depth (relative to the cortical surface) and position (in the anteriorposterior dimension) that could reasonably correspond to 3b. Finally, the pattern of penetrations in and near the central sulcus was reconstructed histologically (see below) (Fig. 2). Because the penetrations were confined to a rigid grid pattern (Crist Instrument Company) at a set angle, the tracks of individual electrode penetrations that struck area $3 \mathrm{~b}$ could be defined, and using the depth of the recording sites within that penetration, area $3 \mathrm{~b}$ sites could be differentiated from overlying area 1 sites; this was the key issue, given that many of the penetrations traversed area 1.

Functional positioning of the electrode array. We monitored the responses to electrical stimulation of the median nerve and to mechanical stimulation of digits $\mathrm{d} 2-\mathrm{d} 5$ at all electrode contacts while advancing the electrode. Although deviation from an ideal penetration angle, as was encountered in this study because of the anatomy of the subjects, violates the formal assumptions necessary for using one-dimensional CSD as a quantitative index of the laminar profile of transmembrane current flow, qualitative assessment of the local field potential and derivative CSD profiles, in conjunction with the colocated MUA profile, were nonetheless extremely valuable in helping to position the electrode array so that it bracketed the cortical region of interest. This approach is illustrated in Figure 1.

Calibration of stimulus intensity. After the electrode was localized to area $3 \mathrm{~b}$, intensity of the tactile stimulus was calibrated by modulating the pressure of the air pulse delivered to the diaphragm at the digit pad. By parametrically varying stimulus intensity during recording of the evoked response, we determined the minimum intensity at which an electrophysiological response was elicited. Thus, the stimulus intensity applied during our experimental conditions was calibrated based on the measured electrophysiological response and not based on a behavioral response indicating sensation by the animal. The stimulus intensity used resulted in a minimal skin indentation barely perceptible by the observer.

Identification of the preferred digit representation at a cortical location. After electrode placement within area $3 \mathrm{~b}$ and calibration of stimulus intensity, the stimulus-evoked response was recorded during tactile stimulation of each digit. The preferred digit representation for a given electrode position was then determined based on the magnitude of the MUA response elicited by stimulation of each digit. If stimulation of one digit produced an MUA response at least $200 \%$ of the responses to stimulation of other digits, we determined that digit to be the preferred representation at this cortical location.

Choice of dependent measures. Our foremost concern in planning electrode penetrations and recording sites was to assure sufficiently high-density recording across the digit representations within layer 4 of area $3 b$. This is because our scientific question centers on activity occurring at the first stage of cortical processing, receiving direct thalamocortical afferents from ventral posterior lateral nucleus (VPL). Our choice of physiologic measure was secondary and constrained by the anatomical features of our preparation. The anatomy of the macaque central sulcus poses a significant obstacle to penetration orthogonal to the area $3 \mathrm{~b}$ layers; it is mostly buried within the central sulcus and its lamination pattern lies at an angle well away from the vertical stereotaxic plane. In light of this anatomic constraint, and since a strictly valid CSD analysis requires that electrode contacts be arrayed along an axis perpendicular to the cortical layers (Mitzdorf, 1985), it was not practical to target the penetrations so that each one, or even most, entered area $3 \mathrm{~b}$ at an angle appropriate for a formally valid interpretation of the CSD profile. Rather, we chose MUA, as a measure of action potential activity, as the primary dependent measure for analysis of the hand representation. This practical choice was facilitated by the fact that individual electrode channels could be confirmed within layer 4 of area $3 \mathrm{~b}$ even when the angle of penetration was oblique with respect to the cortical laminae.

Recording under general anesthesia. To address the concern that heterotopic modulation of digit representation reflects an attention effect, we performed a subset of the recording sessions under general anesthesia. All anesthetized experiments were performed under the close supervision of a veterinarian. Before electrode placement, a 20 gauge intravenous catheter was placed in the saphenous vein under sterile conditions. Lactated Ringer's solution was infused at $15 \mathrm{ml} \cdot \mathrm{kg}^{-1} \cdot \mathrm{h}^{-1}$. Electrode placement then commenced and recordings were completed with the animal fully conscious. After recordings were complete, propofol was infused intravenously at $0.4 \mathrm{mg} \cdot \mathrm{kg}^{-1} \cdot \mathrm{min}^{-1}$ through the intravenous catheter using an infusion pump. Strict aseptic precautions were observed when handling, mixing, and administering propofol. As the animal became somnolent, physiologic monitoring was commenced including continuous monitoring of heart rate, oxygen saturation, blood pressure (noninvasive cuff), respiratory rate, and end-tidal $\mathrm{CO}_{2}$ (via nasal cannula). Temperature was also monitored via rectal probe. A warm air blanket (Bair Hugger System; Arizant) was used to maintain body temperature under anesthesia. Depth of anesthesia was assessed and monitored via the eyeblink and gag reflexes as well as responsiveness to stimulation of the foot. Once the animal was fully anesthetized, a plastic oral airway was placed to prevent prolapse of the tongue and occlusion of the oropharyngeal airway. The animal was kept under constant visual observation using a remote controlled video camera. All experimental conditions performed before anesthesia were then repeated without 
change in the electrode position. At the conclusion of the recording session, propofol was discontinued and the infusion of lactated Ringer's solution continued. As the animal awakened, physiologic monitoring, the airway, and the intravenous catheter were discontinued. The head post fixation and all restraints were released before the animal awakened to allow emergence from anesthesia in a natural unrestrained position. The animal was monitored and warmed continuously until fully awake.

Stimulus conditions and quantification of responses. During each recording session, each digit, $\mathrm{d} 2-\mathrm{d} 4$, contralateral to the electrode was stimulated individually. Additionally, all possible combinations of paired contralateral digits were tested with simultaneous stimulation. Trials were arranged in random order. Each averaged MUA response was quantified by measuring the area under the MUA curve (in microvolts) between 5 and $50 \mathrm{~ms}$ after the stimulus.

Statistical analysis and data presentation. Response to each stimulus condition was compared for all recordings from area $3 \mathrm{~b}$ with determination of a statistically significant response using a two-tailed $t$ test. Before our analyses, we tested the distribution of measurements under a given condition for normality using the nonparametric Kolmogorov-Smirnov test. Distributions were considered normally distributed at a significance level of 0.05 . For assessment of interaction effects, the numerical sum of the area under the MUA curves for separate stimulation of individual digits was compared with the area under the MUA curve for the interaction condition (simultaneous stimulation). This allowed assessment of the degree to which interaction effects were additive versus nonadditive (subadditive or superadditive). Variability of the absolute electrophysiological response magnitude is an inherent feature of awake intracranial recordings in macaques. In our study, the responses (area under the MUA curve) to stimulation of the preferred digit, for example, ranged from 50 to $442 \mu \mathrm{V}$-ms across 28 penetrations in two animals. To meaningfully characterize responses and avoid dominance of single observations (from experiments in which absolute amplitudes are large or small), we normalize the responses derived from each electrode location. For each interaction case (see Figs. 6-8), mean response magnitude \pm SD is scaled and expressed as a percentage of the response to stimulation of the preferred digit. Thus, the preferred digit condition is considered $100 \%$, with no variance, in each figure.

Histology. At the conclusion of data collection, one monkey was killed by a lethal dose of pentobarbital and perfused transcardially with $0.3 \mathrm{~L}$ of saline followed by $3 \mathrm{~L}$ of ice-cold $3 \%$ paraformaldehyde in $0.1 \mathrm{~m}$ phosphate buffer and $1 \mathrm{~L}$ each of 10,20 , and then $30 \%$ sucrose in phosphate buffer. The second monkey went on to serve as a subject in an unrelated auditory cortex experiment. After 1 week in buffered 30\% sucrose, the brain was cut frozen on a sliding microtome at $80 \mu \mathrm{m}$ thickness. The frontal and temporal poles were frozen to the microtome stage, so that the plane of section was approximately perpendicular to the axis of the sylvian fissure. During sectioning, a video camera mounted over the tissue was used to record the appearance and location of every section. Video images were stored in consecutive order on a computer, and later used for three-dimensional reconstruction of the brain volume (Fig. 2).
For reconstruction, every sixth consecutive video image was manually outlined, and volume rendering was done using the volume J plug-in of ImageJ software (Rasband, 1996-2007; Abràmoff and Viergever, 2002). Electrode penetration sites were identified microscopically in Nisslstained and immunolabeled sections and their locations were drawn at corresponding locations on the video images and three-dimensional reconstructions. Individual sites were identified by comparison of their distribution with the penetration coordinates in the electrode guide matrix. Additionally, one site in each hemisphere was marked, 2 weeks before killing, by depositing retrograde tracer through a fine cannula incorporated into the multielectrode [described previously (Lakatos et al., 2005; Smiley et al., 2007)]. Nissl staining with thionin was done on every 12 th consecutive $80-\mu \mathrm{m}$-thick section. Adjacent series of sections were immunolabeled using mouse anti-parvalbumin (Sigma-Aldrich), mouse anti-neurofilament antibody SMI-32 (Sternberger Monoclonals), and mouse anti-neuronal nuclear protein (Millipore Bioscience Research Reagents). Characteristically dense parvalbumin immunostaining was used to delineate layer III/IV in area 3b. 


\section{Results}

This analysis is based on data from 38 separate recording sites within layer 4 of the hand representation of area $3 \mathrm{~b}$, in two rhesus macaques.

\section{Digit maps in area $3 b$}

For each multielectrode recording that bracketed area $3 \mathrm{~b}$, we selected the optimal layer 4 recording based on its location in the middle depth of the cortical laminar profile, the presence of relatively high spontaneous firing rate, and the degree of digit selectivity relative to simultaneous recordings from more superficial and deeper (supragranular and infragranular) sites. To illustrate the application of the last criterion, concurrent MUA recordings from layer 4 and from supragranular and infragranular locations are shown in Figure 3; superimposed traces depict responses to stimulation of each of the digits (d2, d3, d4, or d5). The location of digit representations was always consistent with the expected medial to lateral arrangement known to characterize area $3 \mathrm{~b}$, with $\mathrm{d} 2$ most lateral and d5 most medial (Powell and Mountcastle, 1959b).

Individual digit responses are better isolated in layer 4 than in extragranular layers. However, we found that even within layer 4 the digit map was less discrete than has been previously described (Powell and Mountcastle, 1959b; Poggio and Mountcastle, 1963; Paul et al., 1972; Sur et al., 1982). Although stimulation of individual digits identified a single preferred digit in each case, a clear MUA response was detected after stimulation of any individual digit, regardless of the cortical digit representation being sampled (Fig. 3).

\section{Could the nondiscrete digit map be an artifact of stimulus spread?}

Given that we were unable to define truly discrete digit representations in area $3 b$, it was imperative to confirm that our tactile stimulus was in fact confined to a single digit. We used two control conditions to be certain that mechanical stimulation was confined to the intended digit pad. First, stimulation of individual digits was performed while making measurements of skin displacement at adjacent digits. The stimulus device was placed at each of three adjacent digits $(\mathrm{d} 2-\mathrm{d} 4) . \mathrm{d} 2$ was then stimulated while pressure at $\mathrm{d} 3$ and $\mathrm{d} 4$ was monitored. Figure 4 shows that, when a 45 $\mathrm{mmHg}$ stimulus was applied to $\mathrm{d} 2$, no stimulus was detected at $\mathrm{d} 3$ and $\mathrm{d} 4$. This stimulus magnitude exceeds the stimulus intensity used during the recordings for this study by a factor of 9 or more.

As a second control experiment, we tested the effects of stimulation during anesthetic nerve block of the preferred digit. The rationale was that neurally mediated responses to stimulation of a nonpreferred digit (e.g., stimulation of $\mathrm{d} 3$ while recording from a cortical site with a strong $\mathrm{d} 2$ preference) would persist during nerve block of the preferred digit, indicating direct neural transmission from receptors in the nonpreferred digit to cortex. However, artifactual responses, because of inadvertent spread of stimulation to receptors in the preferred digit, would be suppressed by nerve block of that digit. In addition to the mechanical stimulus, we also used electrical stimulation of the digit pad, as this eliminates mechanical artifacts. Comparison of the response to electrical stimulation of the preferred digit before and after nerve block, demonstrates complete suppression of the MUA response after nerve block, even when the stimulus intensity is increased by a factor of 6 (Fig. $5 A$ ). The response to electrical stimulation of the adjacent (nonpreferred) digit is unchanged after nerve block of the preferred digit (Fig. 5B). Similarly, the response to mechanical stimulation of the preferred digit is attenuated after nerve block (Fig. $5 C$ ), with no change in the response
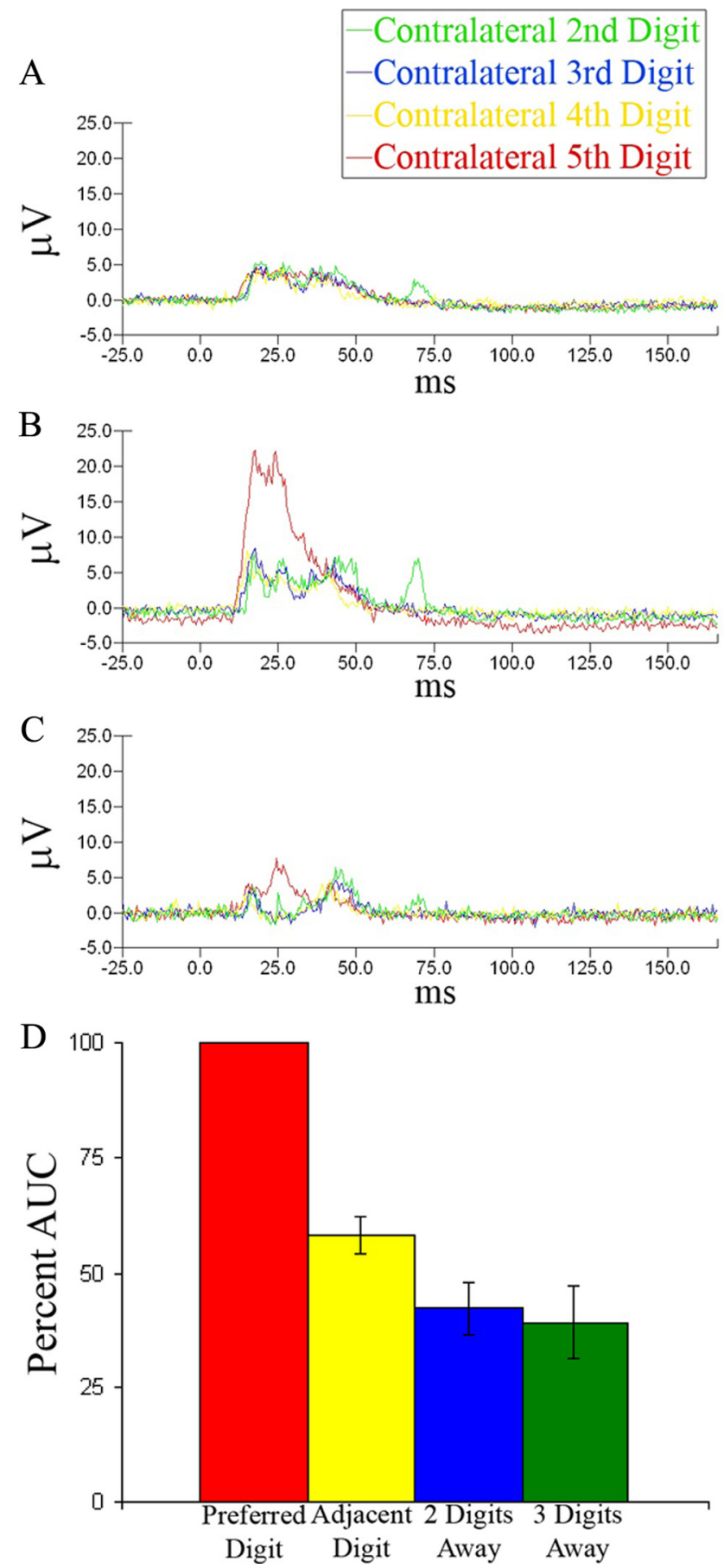

Figure 3. Physiologic mapping of the digit representations in area $3 b$. MUA from contacts in supragranular $(\boldsymbol{A})$, granular (layer 4$)(\boldsymbol{B})$, and infragranular $(\boldsymbol{C})$ cortical layers recorded from a single penetration through the $\mathrm{d} 5$ representation, during independent stimulation of each digit, $\mathrm{d} 2-\mathrm{d} 5$, shows preferential response to $\mathrm{d} 5$ (red tracing) in layer 4 , but not in supragranular or infragranular layers. The stimulus is applied at time $=0$. Quantification of the effects (area under the MUA curve $\pm S D$ ) across all area $3 \mathrm{~b}$ penetrations $(\boldsymbol{D})$ shows a significantly greater response to $\mathrm{d} 5$ stimulation relative to any of the other digits ( $\mathrm{d} 2-\mathrm{d} 4)$, but no significant difference between the nonpreferred digits.

to stimulation of the nonpreferred digit (Fig. 5D). Combining data across all experiments ( $n=6$ for each stimulus type, electrical and mechanical), change in MUA (area under the curve) is significant $(p=0.008$ for electrical stimulation and $p=0.02$ for mechanical stimulation) for the preferred digit when comparing pre- and post-nerve block conditions, but not for the nonpre- 
ferred digit ( $p=0.86$ for electrocutaneous stimulation and $p=$ 0.34 for mechanical stimulation). In light of the aggregate pattern of these findings, we consider it likely that the responses to stimulation of a nonpreferred digit truly reflect "heterotopic" projection from receptors in the nonpreferred digit to the cortical representation of the preferred digit in area $3 \mathrm{~b}$.

\section{Interaction of adjacent and preferred digit stimulation}

Response magnitude (area under the MUA curve) elicited by stimulation of a nonpreferred digit was always lower than that of the preferred digit (Fig. 6). The distribution of measurements within each condition was normally distributed (KolmogorovSmirnov value of $p>0.05$ ). Simultaneous stimulation of the preferred digit and an immediately adjacent digit (e.g., $\mathrm{d} 2+\mathrm{d} 3$; $n=38$ ) yielded a response significantly smaller than the numerical sum of the individual digits, indicating a suppressive interaction effect $(p<0.00000002)$. Similar suppressive interaction effects were detected when comparing the preferred digit with noncontiguous digits [two digits away $(n=25$; e.g., $\mathrm{d} 2+\mathrm{d} 4 ; p<$ $0.00002)$ or three digits away $(n=14$; e.g., $\mathrm{d} 2+\mathrm{d} 5 ; p<0.0005)]$. The response to stimulation of the noncontiguous digit alone was significantly lower than that to stimulation of the adjacent digit alone. However, differences between responses evoked from digits separated from the preferred digit by one or two intervening digits were not significant. Nonetheless, the magnitude of the response to simultaneous stimulation of the preferred plus a nonpreferred digit was similar for all digit combinations and thus did not vary based on distance of the second digit from the preferred digit. This finding implies that the interaction effects result in a broad flat surround. Interestingly, for the $\mathrm{d} 3$ and $\mathrm{d} 4$ representation recordings (12 experiments), we had the opportunity to examine the effect of simultaneous stimulation of the two digits surrounding the preferred digit (Fig. 7), without actual stimulation of the preferred digit itself. For these trials, the interaction condition (two surrounding digits) elicited a smaller magnitude response than their sum indicating a suppressive interaction $(p<0.0001)$.

\section{Are the interaction effects a function of attention or arousal?}

Although stimulation was totally noncontingent, and monkeys were thoroughly habituated to the stimuli, it is possible that the effects we describe result from the fact that stimulation of multiple digits is more arousing, or draws the monkey's attention to a greater extent than stimulation of a single digit. We addressed this possibility by repeating the experimental conditions with the animals in a state under which attention could not play a role. In 14 of the total of 38 experiments, recording was repeated under general anesthesia using intravenously administered propofol, within the same recording session as the awake recordings. Anesthesia was induced with the electrode in place to ensure that recording occurred at the same location before and after the animal was anesthetized. The exact same stimulus and recording conditions and parameters were used before and after administration of the anesthetic. The overall magnitude of the evoked response was lower under anestheof the preferred digit.

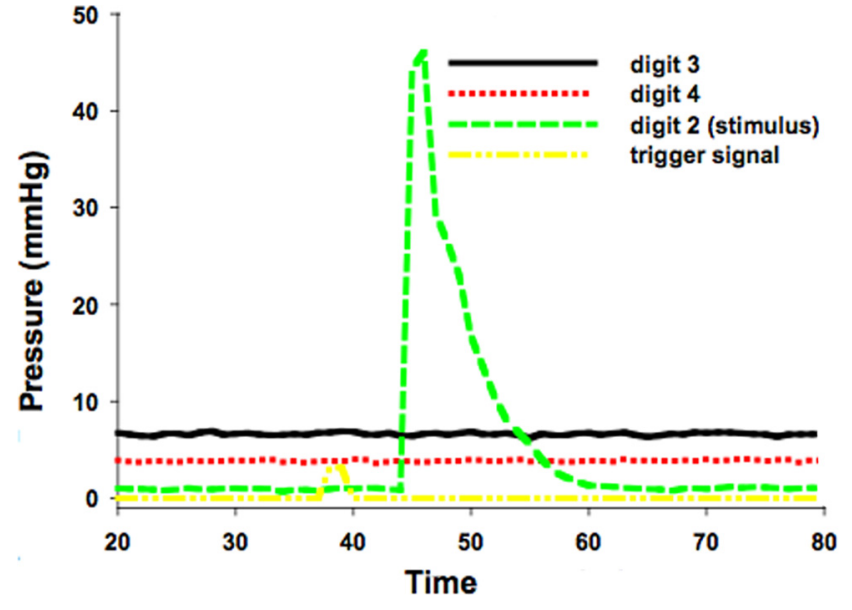

Figure 4. Confirming stimulus isolation using strain gauge measurements. A pressure pulse is applied to $\mathrm{d} 2$ (green) while pressure is measured at $\mathrm{d} 3$ (black) and $\mathrm{d} 4$ (red). Also shown is the trigger that initiated the event (yellow). Time between trigger and onset of the stimulus is 25 $\mathrm{ms}$. Note the slight positive pressure differential applied to $\mathrm{d} 3$ and $\mathrm{d} 4 \mathrm{t}$ to increase the likelihood of detecting a response if it occurred. Pressure is applied to the stimulus device at the digit pad for $5 \mathrm{~ms}$. Lag in decline of pressure measured at $\mathrm{d} 2$ reflects the time required for complete deflation of the stimulus device. The figure shows a single trial, and each point on the horizontal axis is an average of $5 \mathrm{~ms}$.

Figure 5. Confirming stimulus isolation using local anesthesia of the preferred digit. Responses to electrocutaneous $(\boldsymbol{A}, \boldsymbol{B})$ and mechanical $(\boldsymbol{C}, \boldsymbol{D})$ stimulation are shown. After anesthesia of the preferred digit, the MUA response to stimulation of the preferred digit is suppressed $(\boldsymbol{A}, \boldsymbol{C})$. The adjacent digit evokes a similar response $(\boldsymbol{B}, \boldsymbol{D})$ (no significant difference) before and after anesthesia

sia, consistent with previous studies (Gardner and Costanzo, 1980), and responses to stimulation of individual digits showed a greater degree of digit preference with lower amplitude responses to nonpreferred digits. Nonetheless, MUA responses were detected during stimulation of nonpreferred digits. Interaction effects were also preserved under anesthesia. As shown in Figure 8, the response to simultaneous stimulation of adjacent digits is less than the sum of responses to separate stimulation of the digits. This interaction effect was significant $(p<0.005)$.

\section{Discussion}

\section{Dynamic rather than discrete representation of the digits in} area $3 b$ ?

The finding that each "digit representation" in macaque cortical area $3 \mathrm{~b}$ interacts with each of the other digits, is consistent with 

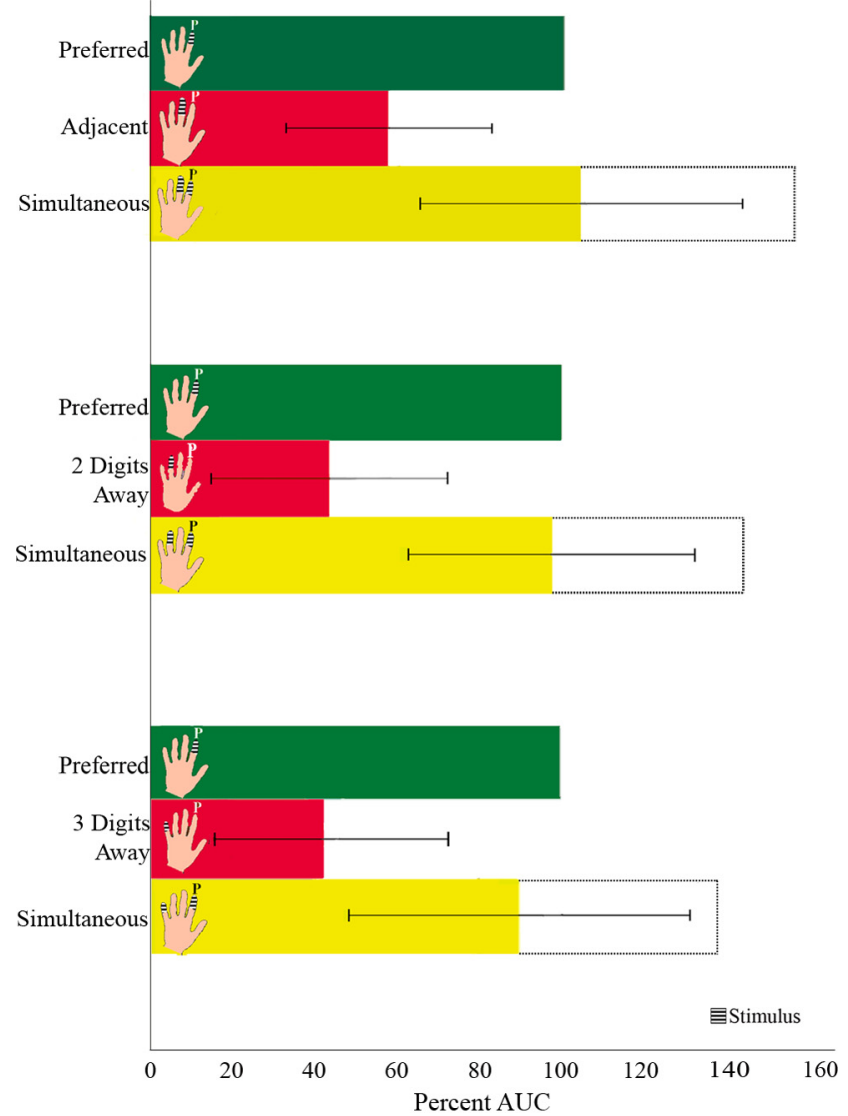

Figure 6. Interaction elicited by stimulation of the preferred digit and one other digit on the same hand. All recordings were made at the same electrode location within the area $3 \mathrm{~b}$ cortical representation of the preferred digit. Although stimulation of the adjacent digit (red) elicits a robust response within the representation of the preferred digit, simultaneous stimulation of both preferred and adjacent digits (yellow) does not elicit any greater response than stimulation of the preferred digit alone (green). Although the magnitude of the response to stimulation of individual adjacent digits (red) declines with distance from the preferred digit (green), note that no significant decrement in the response to simultaneous stimulation (yellow) occurs. Measurements are presented as area under the MUA curve \pm SD, scaled as a percentage of the response to stimulation of the preferred digit (green, 100\%). The sum of individual responses to stimulation of the adjacent and preferred digits is shown as a dotted line. The digit adjacent to $(n=38)$, two digits away $(n=25)$, and three digits away $(n=14)$ from the preferred digit were studied.

recent reports based on experiments in rodents (Nicolelis et al., 1998; Moore et al., 1999; Andermann and Moore, 2006), raccoons (Greek et al., 2003), monkeys (Gardner and Costanzo, 1980; Chen et al., 2003; Reed et al., 2008), and humans (Buchner et al., 1999; Noppeney et al., 1999; Braun et al., 2000, 2002; Iguchi et al., 2001; Jones et al., 2007). Nonetheless, because our findings diverge from the previous impressions of more discrete mapping of the individual digits in primate area $3 \mathrm{~b}$ (Powell and Mountcastle, 1959b; Jones and Powell, 1969; Paul et al., 1972; Sur et al., 1982; Pons et al., 1987; Kaas, 1993), we used several means to ensure that recording was made in area $3 \mathrm{~b}$ and not in an adjacent portion of S1, such as areas 1 or 2, which are known to have broader receptive fields and to integrate inputs from multiple digits (Iwamura, 1998). We also implemented several control procedures to rule out artifacts attributable to spread of stimulation from intended to unintended digit pads.

One potential explanation for the discrepancy between more recent studies including our own and previous studies is the conditions under which recordings were made. Early studies that established the

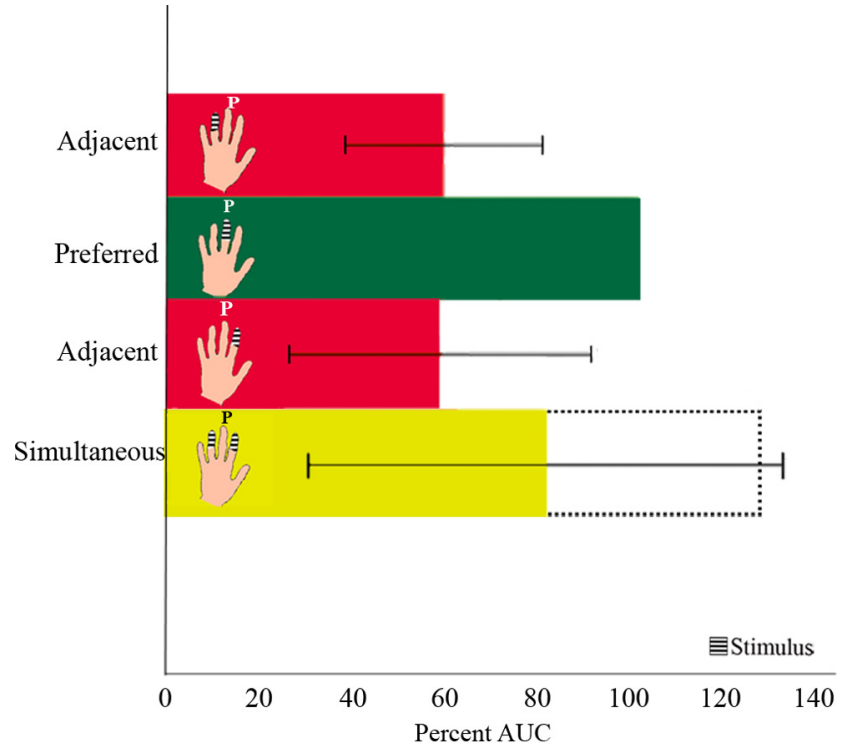

Figure 7. Interaction effect elicited by stimulation two digits surrounding the preferred digit. All recordings were made at the same electrode location within the area $3 \mathrm{~b}$ cortical representation of the preferred digit. Simultaneous stimulation of two digits adjacent to the preferred digit (yellow; $n=12$ ) yields a significant suppression of the response relative to the sum of the two individual digit responses (dotted line). For reference, the preferred digit response (green) and the individual adjacent digit responses (red) are shown. Measurements are presented as area under the MUA curve \pm SD, scaled as a percentage of the response to stimulation of the preferred digit (green, 100\%).

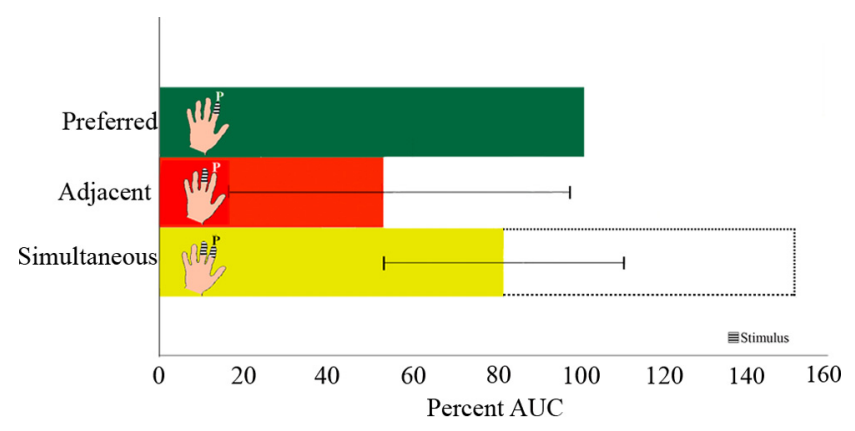

Figure 8. Interaction elicited by simultaneous digit stimulation persists under propofol anesthesia. All recordings were made at the same electrode location within the area $3 \mathrm{~b}$ cortical representation of the preferred digit. The response $(n=14)$ to simultaneous stimulation of adjacent and preferred digits (yellow) is lower magnitude than the preferred digit response alone (green), which is thus not augmented by additional stimulation of the adjacent digit (red). The sum of individual responses to stimulation of the adjacent and preferred digits is shown as a dotted line. Thus, the interaction effect persists even while the animal is under general anesthesia. Measurements are presented as area under the MUA curve \pm SD, scaled as a percentage of the response to stimulation of the preferred digit (green, 100\%).

view of a discrete digit somatotopy in macaques were performed in anesthetized animals (Powell and Mountcastle, 1959b; Jones and Powell, 1969; Paul et al., 1972; Sur et al., 1982; Pons et al., 1987; Kaas, 1993). Although early investigators understood that anesthesia did not alter the distribution of cortical evoked responses or the somatotopic map (Woolsey et al., 1942), it has been known for some time that responsiveness of $\mathrm{S} 1$ neurons in monkeys is compromised by anesthetic effects, altering or masking phenomena that are detected in conscious animals (Gardner and Costanzo, 1980). Our findings (see also Gardner and Costanzo, 1980; Chen et al., 2003; Reed et al., 2008) suggest that this is a partial explanation at best. Our studies were performed in both anesthetized and in fully conscious animals free of the effects of anesthesia. The digit representation does appear 
more discrete under anesthesia, but responses to nonpreferred digits and interactions attributable to stimulation of multiple digits persist in the anesthetized state.

Another potential explanation for the differences between the present and previous findings relates to recording technique. Some previous studies of somatotopy in area $3 \mathrm{~b}$ measured action potentials from single units (Powell and Mountcastle, 1959b), whereas we measured multiunit action potential activity. Our controls for specificity of stimulation (above) do not address the specificity of multiunit recordings. However, we note that numerous previous studies that used multiunit recordings along with single-unit recording also suggested a discrete representation (Pons et al., 1992; Kaas, 1993). Moreover, our previous studies using electrodes of the type used here suggest clear differentiation of even very large paroxysmal bursts with intercontact spacing as low as $75 \mu \mathrm{m}$ (Schroeder et al., 1990, 1997). Finally, the nature of the interaction effects we observe is mostly suppressive. If our findings were attributable to a limitation of the specificity of the recording technique (i.e., the electrodes could not isolate a single finger representation), the predicted effects would be simply additive and excitatory rather than suppressive. Thus, it is safe to conclude that there are indeed interactions between the digit representations in area $3 \mathrm{~b}$.

\section{Potential mechanisms supporting the interaction effects}

Interactions between the digit representations are likely to result, at least in part, from the nature of the thalamocortical afferents. For example, projections from VPL reach regions of cortex significantly larger (perhaps by a factor of 3 or more) than individual digit representations (Garraghty and Sur, 1990). Thus, activation of single thalamocortical afferents will provide input to regions of cortex that represent more than one digit. Although integration of these overlapping inputs could be a manifestation of selective suppression and augmentation of inputs at the intracortical level, it is important to consider the possibility of subcortical modulation as a contributor to the ultimate interaction effects. In rodent whisker barrel cortex, for example, although it had long been thought that cortical interneurons were central to integration of inputs from multiple whiskers, recent work has suggested that subcortical integration plays an important role (Higley and Contreras, 2007). Presynaptic or postsynaptic inhibition as well as amplification could operate at either or a combination of these levels. Cortical interneurons in area $3 \mathrm{~b}$, for example, could modulate thalamocortical inputs, with the balance between the strength of thalamocortical input and the magnitude of intracortical inhibition determining the response. This explanation of our findings would be consistent with that posited by Gardner and Costanzo (1980) to explain neural occlusion detected in their studies of monkey S1. Alternatively, postsynaptic inhibition of VPL neurons could contribute to or dominate the sculpting of cortical responses.

Future study toward delineating the locus of modulatory effects that support interdigit interactions within area $3 \mathrm{~b}$ could be addressed by thalamic recordings as well as by detailed assessment of differential responses within layer 4 compared with those arising in extragranular layers. Although some of our recordings suggested response differences across layers, our experiments were not designed to address this question and it must be deferred to future study.

\section{Functional significance of suppressive interactions?}

Psychophysical studies of human perception indicate that subjects are unable to perceive a stimulus applied to one finger and simultaneously ignore a different stimulus presented to another finger (Evans and Craig, 1991). Although it is clear that monkeys and humans use multiple digits at once in the course of haptic exploration of shape and in the performance of complex manual tasks, the si- multaneous use of multiple digit pads does not enhance and may in fact impede the efficiency of tactile discrimination (Craig, 1985; Loomis et al., 1991). Our studies do not directly address the issue of perception. However, the suppressive interaction effects we describe might represent a physiologic mechanism for equalizing tactile sensitivity at all involved receptor surfaces. By minimizing the distraction of variable sensation across multiple digits in this manner, area 3b might be optimized for haptic exploration or a concerted manual task. This can be understood as a means for enhancing the signal-tonoise of the sensory system to favor, for example, proprioceptive as opposed to tactile perceptual acuity, the concept of "sensory inhibition” proposed by Georg Von Békésy (Von Békésy, 1967) for the understanding of sensory systems.

Chen et al. (2003) described a hemodynamic correlate of the well established phenomenon of tactile funneling (Von Békésy, 1963; Gardner and Spencer, 1972), using optical imaging. Whereas separate stimulation of two individual digits manifested as distinct hemodynamic responses in area $3 \mathrm{~b}$, a single response, shifted to the interface between the individual responses was elicited by simultaneous stimulation of the two adjacent digits. Our studies were not designed to address the interaction effect explored by Chen and colleagues and our sampling across the cortical surface was restricted to $1 \mathrm{~mm}$ increments by the electrode guide matrix that we used. However, our finding of an interaction effect in response to simultaneous stimulation of adjacent digits, with relative suppression of the response within the digit representation, may depict a part of the funneling response observed by Chen et al. (2003): suppression of the individual digit representation during simultaneous stimulation. Moreover, we can note anecdotally that, in two cases, our electrode penetrated area $3 \mathrm{~b}$ at an oblique angle and traversed adjacent finger representations in layer 4 . In these cases, during simultaneous stimulation of the two digits, we detected a mild excitatory interaction at the electrode contact located at the interface between the digit representations.

In conclusion, we have demonstrated a dynamic representation of the digits in macaque area $3 \mathrm{~b}$ under normal physiologic conditions. The interaction effects we observe may represent a physiologic substrate optimized to meet specific perceptual demands with preparatory alteration in the map triggered by contextual factors, such as the number of digits stimulated simultaneously. This concept of the hand representation holds promise for further understanding the processing in primary somatosensory cortex of perceptual phenomena at variance with more rigid models of hand somatotopy. Additional study using electrical recordings targeted based on highresolution functional MRI and optical imaging holds much promise for addressing such brain-perception paradoxes.

\section{References}

Abràmoff MD, Viergever MA (2002) Computation and visualization of three-dimensional soft tissue motion in the orbit. IEEE Trans Med Imaging 21:296-304.

Allard T, Clark SA, Jenkins WM, Merzenich MM (1991) Reorganization of somatosensory area $3 \mathrm{~b}$ representations in adult owl monkeys after digital syndactyly. J Neurophysiol 66:1048-1058.

Andermann ML, Moore CI (2006) A somatotopic map of vibrissa motion direction within a barrel column. Nat Neurosci 9:543-551.

Braun C, Wilms A, Schweizer R, Godde B, Preissl H, Birbaumer N (2000) Activity patterns of human somatosensory cortex adapt dynamically to stimulus properties. Neuroreport 11:2977-2980.

Braun C, Haug M, Wiech K, Birbaumer N, Elbert T, Roberts LE (2002) Functional organization of primary somatosensory cortex depends on the focus of attention. Neuroimage 17:1451-1458.

Buchner H, Reinartz U, Waberski TD, Gobbelé R, Noppeney U, Scherg M (1999) Sustained attention modulates the immediate effect of de- 
afferentation on the cortical representation of the digits: source localization of somatosensory evoked potentials in humans. Neurosci Lett 260:57-60.

Burton H, Sinclair RJ (1996) Somatosensory cortex and tactile perceptions. In: Pain and touch (Kruger L, ed), pp 105-177. San Diego: Academic.

Chen LM, Friedman RM, Roe AW (2003) Optical imaging of a tactile illusion in area $3 \mathrm{~b}$ of the primary somatosensory cortex. Science 302:881-885.

Clarey JC, Tweedale R, Calford MB (1996) Interhemispheric modulation of somatosensory receptive fields: evidence for plasticity in primary somatosensory cortex. Cereb Cortex 6:196-206.

Coq JO, Xerri C (1999) Acute reorganization of the forepaw representation in the rat SI cortex after focal cortical injury: neuroprotective effects of piracetam treatment. Eur J Neurosci 11:2597-2608.

Craig JC (1985) Attending to two fingers: two hands are better than one. Percept Psychophys 38:496-511.

Evans PM, Craig JC (1991) Tactile attention and the perception of moving tactile stimuli. Percept Psychophys 49:355-364.

Friedman RM, Chen LM, Roe AW (2008) Responses of areas $3 \mathrm{~b}$ and 1 in anesthetized squirrel monkeys to single- and dual-site stimulation of the digits. J Neurophysiol 100:3185-3196.

Gardner EP, Costanzo RM (1980) Spatial integration of multiple-point stimuli in primary somatosensory cortical receptive fields of alert monkeys. J Neurophysiol 43:420-443.

Gardner EP, Spencer WA (1972) Sensory funneling. I. Psychophysical observations of human subjects and responses of cutaneous mechanoreceptive afferents in the cat to patterned skin stimuli. J Neurophysiol 35:925-953.

Garraghty PE, Sur M (1990) Morphology of single intracellularly stained axons terminating in area $3 \mathrm{~b}$ of macaque monkeys. J Comp Neurol 294:583-593.

Greek KA, Chowdhury SA, Rasmusson DD (2003) Interactions between inputs from adjacent digits in somatosensory thalamus and cortex of the raccoon. Exp Brain Res 151:364-371.

Higley MJ, Contreras D (2007) Cellular mechanisms of suppressive interactions between somatosensory responses in vivo. J Neurophysiol 97:647-658.

Iguchi Y, Hoshi Y, Hashimoto I (2001) Selective spatial attention induces short-term plasticity in human somatosensory cortex. Neuroreport 12:3133-3136.

Iwamura Y (1998) Hierarchical somatosensory processing. Curr Opin Neurobiol 8:522-528.

Jenkins WM, Merzenich MM (1987) Reorganization of neocortical representations after brain injury: a neurophysiological model of the bases of recovery from stroke. Prog Brain Res 71:249-266.

Jones EG, Powell TP (1969) Connexions of the somatic sensory cortex of the rhesus monkey. II. Contralateral cortical connexions. Brain 92:717-730.

Jones SR, Pritchett DL, Stufflebeam SM, Hämäläinen M, Moore CI (2007) Neural correlates of tactile detection: a combined magnetoencephalography and biophysically based computational modeling study. J Neurosci 27:10751-10764.

Kaas JH (1993) The functional organization of somatosensory cortex in primates. Ann Anat 175:509-518.

Lakatos P, Shah AS, Knuth KH, Ulbert I, Karmos G, Schroeder CE (2005) An oscillatory hierarchy controlling neuronal excitability and stimulus processing in the auditory cortex. J Neurophysiol 94:1904-1911.

Legatt AD, Arezzo J, Vaughan HG Jr (1980) Averaged multiple unit activity as an estimate of phasic changes in local neuronal activity: effects of volume-conducted potentials. J Neurosci Methods 2:203-217.

Loomis JM, Klatzky RL, Lederman SJ (1991) Similarity of tactual and visual picture recognition with limited field of view. Perception 20:167-177.

Mitzdorf U (1985) Current source-density method and application in cat cerebral cortex: investigation of evoked potentials and EEG phenomena. Physiol Rev 65:37-100.

Mogilner A, Grossman JA, Ribary U, Joliot M, Volkmann J, Rapaport D, Beasley RW, Llinás RR (1993) Somatosensory cortical plasticity in adult humans revealed by magnetoencephalography. Proc Natl Acad Sci U S A 90:3593-3597.

Moore CI, Nelson SB, Sur M (1999) Dynamics of neuronal processing in rat somatosensory cortex. Trends Neurosci 22:513-520.

Nicholson C, Freeman JA (1975) Theory of current source density analysis and determination of conductivity tensor for anuran cerebellum. J Neurophysiol 38:356-368.

Nicolelis MA, Ghazanfar AA, Stambaugh CR, Oliveira LM, Laubach M,
Chapin JK, Nelson RJ, Kaas JH (1998) Simultaneous encoding of tactile information by three primate cortical areas. Nat Neurosci 1:621-630.

Noppeney U, Waberski TD, Gobbelé R, Buchner H (1999) Spatial attention modulates the cortical somatosensory representation of the digits in humans. Neuroreport 10:3137-3141.

Paul RL, Merzenich M, Goodman H (1972) Representation of slowly and rapidly adapting cutaneous mechanoreceptors of the hand in Brodmann's areas 3 and 1 of Macaca mulatta. Brain Res 36:229-249.

Poggio GF, Mountcastle VB (1963) The functional properties of ventrobasal thalamic neuronsstudied in unanesthetized monkeys. J Neurophysiol 26:775-806.

Pons TP, Wall JT, Garraghty PE, Cusick CG, Kaas JH (1987) Consistent features of the representation of the hand in area $3 \mathrm{~b}$ of macaque monkeys. Somatosens Res 4:309-331.

Pons TP, Garraghty PE, Mishkin M (1992) Serial and parallel processing of tactual information in somatosensory cortex of rhesus monkeys. J Neurophysiol 68:518-527.

Powell TP, Mountcastle VB (1959a) The cytoarchitecture of the postcentral gyrus of the monkey Macaca mulatta. Bull Johns Hopkins Hosp 105:108-131.

Powell TP, Mountcastle VB (1959b) Some aspects of the functional organization of the cortex of the postcentral gyrus of the monkey: a correlation of findings obtained in a single unit analysis with cytoarchitecture. Bull Johns Hopkins Hosp 105:133-162.

Qi H-X, Preuss TM, Kaas JH (2008) Somatosensory areas of the cerebral cortex: architectonic characteristics and modular organization. In: The senses: a comprehensive reference, Vol 6, Somatosensation (Gardner EP, Kaas JH, eds), pp 143-170. London: Elsevier.

Rasband WS (1996-2007) ImageJ: image processing and analysis in JAVA. Bethesda, MD: National Institutes of Health.

Rasmusson DD, Webster HH, Dykes RW (1992) Neuronal response properties within subregions of raccoon somatosensory cortex 1 week after digit amputation. Somatosens Mot Res 9:279-289.

Reed JL, Pouget P, Qi HX, Zhou Z, Bernard MR, Burish MJ, Haitas J, Bonds $\mathrm{AB}$, Kaas JH (2008) Widespread spatial integration in primary somatosensory cortex. Proc Natl Acad Sci U S A 105:10233-10237.

Schroeder CE, Tenke CE, Givre SJ, Arezzo JC, Vaughan HG Jr (1990) Laminar analysis of bicuculline-induced epileptiform activity in area 17 of the awake macaque. Brain Res 515:326-330.

Schroeder CE, Seto S, Arezzo JC, Garraghty PE (1995a) Electrophysiological evidence for overlapping dominant and latent inputs to somatosensory cortex in squirrel monkeys. J Neurophysiol 74:722-732.

Schroeder CE, Steinschneider M, Javitt DC, Tenke CE, Givre SJ, Mehta AD, Simpson GV, Arezzo JC, Vaughan HG Jr (1995b) Localization of ERP generators and identification of underlying neural processes. Electroencephalogr Clin Neurophysiol Suppl 44:55-75.

Schroeder CE, Seto S, Garraghty PE (1997) Emergence of radial nerve dominance in median nerve cortex after median nerve transection in an adult squirrel monkey. J Neurophysiol 77:522-526.

Schroeder CE, Mehta AD, Givre SJ (1998) A spatiotemporal profile of visual system activation revealed by current source density analysis in the awake macaque. Cereb Cortex 8:575-592.

Smiley JF, Hackett TA, Ulbert I, Karmas G, Lakatos P, Javitt DC, Schroeder CE (2007) Multisensory convergence in auditory cortex, I. Cortical connections of the caudal superior temporal plane in macaque monkeys. J Comp Neurol 502:894-923.

Sur M, Nelson RJ, Kaas JH (1982) Representations of the body surface in cortical areas $3 \mathrm{~b}$ and 1 of squirrel monkeys: comparisons with other primates. J Comp Neurol 211:177-192.

Turnbull BG, Rasmusson DD (1990) Acute effects of total or partial digit denervation on raccoon somatosensory cortex. Somatosens Mot Res 7:365-389.

Von Békésy G (1963) Interaction of paired sensory stimuli and conduction in peripheral nerves. J Appl Physiol 18:1276-1284.

Von Békésy G (1967) Sensory inhibition. Princeton: Princeton UP.

Woolsey CN, Marshall WH, Bard P (1942) Representation of cutaneous tactile sensibility in the cerebral cortex of the monkey as indicated by evoked potentials. Bull Johns Hopkins Hosp 70:399-441.

Zarzecki P, Witte S, Smits E, Gordon DC, Kirchberger P, Rasmusson DD (1993) Synaptic mechanisms of cortical representational plasticity: somatosensory and corticocortical EPSPs in reorganized raccoon SI cortex. J Neurophysiol 69:1422-1432. 